\title{
Operator product expansion of the non-local gluon condensate
}

\author{
V.M. Braun, ${ }^{a}$ K.G. Chetyrkin ${ }^{b, c}$ and B.A. Kniehl ${ }^{c}$ \\ ${ }^{a}$ Institut für Theoretische Physik, Universität Regensburg, \\ 93040 Regensburg, Germany \\ ${ }^{b}$ Institut für Theoretische Teilchenphysik, Karlsruhe Institute of Technology (KIT), \\ Wolfgang-Gaede-Straße 1, 76131 Karlsruhe, Germany \\ ${ }^{c}$ II. Institut für Theoretische Physik, Universität Hamburg, \\ Luruper Chaussee 149, 22761 Hamburg, Germany \\ E-mail: vladimir.braun@ur.de, Konstantin.Chetyrkin@kit.edu, \\ kniehl@desy.de
}

ABSTRACT: We consider the short-distance expansion of the product of two gluon field strength tensors connected by a straight-line-ordered Wilson line. The vacuum expectation value of this nonlocal operator is a common object in studies of the QCD vacuum structure, whereas its nucleon expectation value is known as the gluon quasi-parton distribution and is receiving a lot of attention as a tool to extract gluon distribution functions from lattice calculations. Extending our previous study [1], we calculate the three-loop coefficient functions of the scalar operators in the operator product expansion up to dimension four. As a by-product, the three-loop anomalous dimension of the nonlocal two-gluon operator is obtained as well.

KeYwords: Deep Inelastic Scattering (Phenomenology), QCD Phenomenology

ArXiv EPrint: 2103.09478 


\section{Contents}

1 Introduction 1

2 Preliminaries 2

3 Calculation $\quad 3$

4 Results 4

$5 \quad$ RG improvements of $\Pi_{\perp \perp}^{0}$ and $\Pi_{\| \perp}^{0} \quad 7$

6 RG improvements of the CFs 9

$\begin{array}{lll}7 & \text { Conclusions } & 10\end{array}$

\section{Introduction}

In this work, we construct the operator product expansion (OPE) of the non-local twogluon operator

$$
\mathbb{G}_{\mu \nu \alpha \beta}(z)=g^{2} F_{\mu \nu}(z v)[z v, 0] F_{\alpha \beta}(0),
$$

where $g$ is the gauge coupling, $F_{\mu \nu}(x)$ is the gluon field strength tensor, $v^{\mu}$ is an auxiliary four-vector, with $v^{2} \neq 0$, and $z$ is a real number. In addition, $[z v, 0]$ is a straight-lineordered Wilson line connecting the two field strength tensors,

$$
[z v, 0]=\mathcal{P} \exp \left[i g \int_{0}^{z} d z^{\prime} v^{\mu} A_{\mu}\left(z^{\prime} v\right)\right],
$$

with $A_{\mu}(x)$ being the gluon field in the adjoint representation of the color gauge group.

The motivation for this study is twofold. On the one hand, the vacuum expectation value (VEV) of the non-local operator in eq. (1.1), the so-called non-local gluon condensate, describes the correlation of gluon fields in QCD vacuum as a function of their distance and is the basic quantity, e.g., in the stochastic model of the QCD vacuum [2,3]. It also governs the effect of gluon condensation on the mass spectra of heavy quarkonia and the shortdistance expansion of the heavy-quark potential [4-7]. Specifically, it is a central object in nonrelativistic QCD (NRQCD), notably in potential NRQCD (pNRQCD), where its chromo-electric and chromo-magnetic components enter the definitions of the heavy-quark potential from the QCD static energy of a heavy quark-antiquark pair and the gluelump masses, the theoretical treatment of quarkonium hybrids [8], and the determinations of the ultrasoft contribution to the QCD static energy [9] and of long-distrance matrix elements 
of heavy-quarkonium production [10] and decay [11]. Lattice QCD studies of the nonlocal gluon condensate exist aiming at extracting the gluon correlation length [12-14], the strong-coupling constant $\alpha_{s}=g^{2} /(4 \pi)$ via the QCD static energy [15], and also its behavior at the deconfinement phase transition at high temperatures (see, e.g., ref. [16]). A similar two-gluon correlator, albeit with a different Wilson line contour, appears in the definition of the rapidity anomalous dimension (AD), alias Collins-Soper kernel [17].

On the other hand, the nucleon matrix elements of the same non-local operator, usually referred to as gluon quasi parton distribution functions (qPDFs), are attracting increasing interest (see ref. [18] for a review). They can be calculated on the lattice for spacelike separations [19] and matched to the usual collinear gluon parton distribution functions (PDFs) using continuum perturbation theory [20-22]. This technique is attractive, as it allows one to probe the gluon PDF more directly than with other approaches, but it is also challenging. In particular, the renormalization of the non-local gluon operator involves subtleties [23, 24], and also lattice calculations are very challenging due to high statistical noise and the necessity to inject a very large momentum in the nucleon, which requires the use of very fine lattices. Using the ratio of the nucleon to vacuum matrix elements in such calculations can be advantageous [25], as in this way all linear ultraviolet (UV) divergences related to the Wilson line renormalization [26] get canceled.

In this paper, we consider the OPE of the non-local operator in eq. (1.1) to three-loop accuracy, taking into account all scalar operators up to dimension four. As a by-product of this calculation, the three-loop AD (matrix) of the non-local gluon operator is obtained. From the technical point of view, this calculation is an extension of our work in ref. [1], where the perturbative contribution to the OPE was calculated to three-loop accuracy and the two-loop AD was derived. We will mostly adopt the conventions and the notation of ref. [1], a short summary of which is given in section 2. The calculation is described in section 3. The results for the relevant ADs and coefficient functions (CFs) are presented in section 4. The renormalization group (RG) evolution equations for the computed CFs as well as the RG improvement of the purely perturbative contributions are considered in sections 5 and 6 . Section 7 is reserved for a summary and conclusions.

\section{Preliminaries}

The vacuum expectation value (VEV) of the non-local gluon operator in eq. (1.1),

$$
\Pi_{\mu \nu \alpha \beta}(z)=\left\langle 0\left|\mathbb{G}_{\mu \nu \alpha \beta}(z)\right| 0\right\rangle,
$$

can be written in terms of two invariant functions, $\Pi_{\perp \perp}(z)$ and $\Pi_{\| \perp}(z)$, which correspond to contributions with different Lorentz symmetry and do not mix under renormalization [1], as

$$
\begin{aligned}
\Pi_{\mu \nu \alpha \beta}(z)= & \left(g_{\mu \alpha}^{\perp} g_{\nu \beta}^{\perp}-g_{\nu \alpha}^{\perp} g_{\mu \beta}^{\perp}\right) \Pi_{\perp \perp}(z)+\left(g_{\mu \alpha}^{\|} g_{\nu \beta}^{\perp}-g_{\nu \alpha}^{\|} g_{\mu \beta}^{\perp}-g_{\mu \beta}^{\|} g_{\nu \alpha}^{\perp}+g_{\nu \beta}^{\|} g_{\mu \alpha}^{\perp}\right) \Pi_{\| \perp}(z) \\
= & \left(g_{\mu \alpha} g_{\nu \beta}-g_{\nu \alpha} g_{\mu \beta}\right) \Pi_{\perp \perp}(z) \\
& +\frac{1}{v^{2}}\left(v_{\mu} v_{\alpha} g_{\nu \beta}-v_{\nu} v_{\alpha} g_{\mu \beta}-v_{\mu} v_{\beta} g_{\nu \alpha}+v_{\nu} v_{\beta} g_{\mu \alpha}\right)\left[\Pi_{\| \perp}(z)-\Pi_{\perp \perp}(z)\right]
\end{aligned}
$$


where

$$
g_{\mu \nu}^{\|}=\frac{v_{\mu} v_{\nu}}{v^{2}}, \quad g_{\mu \nu}^{\perp}=g_{\mu \nu}-\frac{v_{\mu} v_{\nu}}{v^{2}} .
$$

The renormalization of $\Pi_{\perp \perp}(z)$ and $\Pi_{\| \perp}(z)$ is determined by their respective ADs, $\gamma_{\perp \perp}$ and $\gamma_{\| \perp}$, which are currently known to two-loop accuracy $[1,23,24]$. Notice that the renormalization factors are local, i.e., they do not depend on the distance between the fields. They can be interpreted as the renormalization factors of local "heavy-light" operators in an effective field theory (see refs. [1, 23, 24] for details).

In this work, we consider the OPE in the limit $z \rightarrow 0$ of the invariant functions $\Pi_{\perp \perp}(z)$ and $\Pi_{\| \perp}(z)$ taking into account contributions of the scalar CP-even operators,

$$
O_{2}=\sum_{i} m_{i} \bar{\psi}_{i}(0) \psi_{i}(0), \quad O_{1}=F_{\alpha \beta}(0) F^{\alpha \beta}(0),
$$

where $\psi_{i}$ stands for the $i$-th quark field with mass $m_{i}$. We do not consider operators of mass dimension higher than four. ${ }^{1}$ To this accuracy, we have

$$
\begin{aligned}
& \Pi_{\perp \perp}(z) \underset{z \rightarrow 0}{=} \Pi_{\perp \perp}^{m^{4}}(z)\langle\mathbb{1}\rangle+C_{2}^{\perp \perp}(z)\left\langle 0\left|O_{2}\right| 0\right\rangle+\frac{g^{2}}{12} C_{1}^{\perp \perp}(z)\left\langle 0\left|O_{1}\right| 0\right\rangle, \\
& \Pi_{\| \perp}(z) \underset{z \rightarrow 0}{=} \Pi_{\| \perp}^{m^{4}}(z)\langle\mathbb{1}\rangle+C_{2}^{\| \perp}(z)\left\langle 0\left|O_{2}\right| 0\right\rangle+\frac{g^{2}}{12} C_{1}^{\| \perp}(z)\left\langle 0\left|O_{1}\right| 0\right\rangle,
\end{aligned}
$$

where $\Pi_{\perp \perp}^{m^{4}}(z)$ and $\Pi_{\| \perp}^{m^{4}}(z)$ stand for the purely perturbative contributions expanded in the quark masses through order $m_{q}^{4}$. They can be naturally represented as

$$
\begin{aligned}
& \Pi_{\perp \perp}^{m^{4}}(z)=\frac{C_{0}^{\perp \perp}(z)}{z^{4}}+\frac{C_{m^{2}}^{\perp \perp}(z)}{z^{2}} \sum_{i} m_{i}^{2}+C_{m^{4}, \mathrm{di}}^{\perp \perp}(z) \sum_{i} m_{i}^{4}+C_{m^{4}, \mathrm{nd}}^{\perp \perp}(z) \sum_{i \neq j} m_{i}^{2} m_{j}^{2} \\
& \Pi_{\| \perp}^{m^{4}}(z)=\frac{C_{0}^{\| \perp}(z)}{z^{4}}+\frac{C_{m^{2}}^{\| \perp}(z)}{z^{2}} \sum_{i} m_{i}^{2}+C_{m^{4}, \mathrm{di}}^{\| \perp}(z) \sum_{i} m_{i}^{4}+C_{m^{4}, \mathrm{nd}}^{\| \perp}(z) \sum_{i \neq j} m_{i}^{2} m_{j}^{2}
\end{aligned}
$$

where $C_{\perp \perp}^{0}(z)$ and $C_{\| \perp}^{0}(z)$ correspond to massless, purely perturbative contributions, which are known to two- and three-loop accuracy from refs. [28] and [1], respectively. The new contribution of this work is the calculation of the CFs $C_{m^{2}}^{\perp \frac{\perp}{}}, C_{m^{4}, \mathrm{di}}^{\perp \perp}, C_{m^{4}, \text { nd }}^{\perp \perp}, C_{2}^{\perp \perp}, C_{1}^{\perp \perp}$, $C_{m^{2}}^{\| \perp}, C_{m^{4}, \text { di }}^{\| \perp}, C_{m^{4}, \text { nd }}^{\| \perp}, C_{2}^{\| \perp}, C_{1}^{\| \perp}$, and the ADs $\gamma_{\perp \perp}, \gamma_{\| \perp}$ to three-loop accuracy.

\section{Calculation}

We compute the bare CFs of the operators in eq. (2.4) at the three-loop level using essentially the same techniques as in ref. [1] and the well-known method of projectors [29, 30]. The color factors are evaluated with the help of the FORM [31] package COLOR [32].

Let us briefly discuss the renormalization procedure. The renormalization matrix of the operators in eq. (2.4) has been known for a long time [33-36]. Since the CFs $C_{1}^{\perp \perp}$ and

\footnotetext{
${ }^{1}$ We also do not consider contributions of tensor operators, e.g., $G_{\alpha \xi}(0) G_{\beta}^{\xi}(0)$ (symmetrized over the open indices and with the traces subtracted), which do not contribute to the VEV of the non-local operator, but are relevant for hadron matrix elements. Notice that the corresponding OPE in the tree approximation is known through operators of dimension eight [27].
} 
$C_{1}^{\| \perp}$ are non-zero already in the tree approximation, their proper renormalization requires the knowledge of the $Z$ factors, $Z_{\perp \perp}$ and $Z_{\| \perp}$, at three loops. Thus, the requirement of finiteness of the CFs of the operator $O_{1}$ provides an alternative way for computing $Z_{\perp \perp}$ and $Z_{\| \perp}$. From our results for the bare CFs, $\left(C_{1}^{\perp \perp}\right)_{B}$ and $\left(C_{1}^{\| \perp}\right)_{B}$, we successfully construct the three-loop $Z$ factors, $Z_{\perp \perp}$ and $Z_{\| \perp}$, as well as the corresponding ADs. We also find full agreement with the corresponding two-loop results, first computed in ref. [1].

\section{Results}

In this and the next two sections, we present our results for the case of standard QCD with the $\mathrm{SU}(3)$ gauge group and $n_{f}$ active quarks triplets. The results for the CFs are presented for the case of a spacelike unit vector $v$, with $v^{2}=-1$, and the variable $L_{z}=\ln \left(\mu \mathrm{e}^{\gamma_{E}} z / 2\right)$ set to zero. The missing terms proportional to powers $\left(L_{z}\right)^{i}$ with $i=1,2,3$ can be easily restored with the help of the corresponding evolution equations (see section 6). Full results for a generic gauge group including the momentum/position dependence as well the case of $v^{2}=1$ are appended in the arxiv submission of this paper as ancillary files in a computer readable format.

Expanding a generic $\operatorname{AD} \gamma(a)$ in $a=g^{2} /\left(16 \pi^{2}\right)$ as

$$
\gamma=\sum_{n \geq 1}(\gamma)_{n} a^{n}
$$

our results for the ADs $\gamma_{\perp \perp}$ and $\gamma_{\| \perp}$ read:

$$
\begin{aligned}
& \left(\gamma_{\perp \perp}\right)_{1}=-3, \\
& \left(\gamma_{\perp \perp}\right)_{2}=-34+6 \pi^{2}+\frac{13}{3} n_{f}, \\
& \left(\gamma_{\perp \perp}\right)_{3}=-\frac{899}{2}-10 \pi^{2}+18 \pi^{4}-108 \zeta_{3}+n_{f}\left(76+\frac{16}{3} \pi^{2}-2 \pi^{4}+40 \zeta_{3}\right)+\frac{2}{3} n_{f}^{2}, \\
& \left(\gamma_{\| \perp}\right)_{1}=0, \\
& \left(\gamma_{\| \perp}\right)_{2}=6 \pi^{2}, \\
& \left(\gamma_{\| \perp}\right)_{3}=8 \pi^{2}+18 \pi^{4}+n_{f}\left(\frac{22}{3} \pi^{2}-2 \pi^{4}\right) .
\end{aligned}
$$

Expanding the CFs $C_{m^{2}}, C_{m^{4}, \mathrm{di}}, C_{m^{4}, \text { nd }}, C_{2}$, and $C_{1}$ as

$$
\begin{array}{rlrl}
C_{m^{2}} & =\sum_{n \geq 2}\left(C_{m^{2}}\right)_{n} a^{n}, \quad C_{m^{4}, \mathrm{di}} & =\sum_{n \geq 2}\left(C_{m^{4}, \mathrm{di}}\right)_{n} a^{n}, \quad C_{m^{4}, \mathrm{nd}}=\sum_{n \geq 3}\left(C_{m^{4}, \mathrm{nd}}\right)_{n} a^{n}, \\
C_{2} & =\sum_{n \geq 1} g^{2}\left(C_{2}\right)_{n} a^{n}, & C_{1} & =1+\sum_{n \geq 1}\left(C_{1}\right)_{n} a^{n},
\end{array}
$$

we find the coefficients appearing in eq. (4.3) to be

$$
\begin{aligned}
& \left(C_{m^{2}}^{\perp \perp}\right)_{2}=128 \\
& \left(C_{m^{2}}^{\perp \perp}\right)_{3}=\frac{15872}{3}+\frac{256}{3} \pi^{2}+\frac{128}{45} \pi^{4}+768 \zeta_{3}-\frac{1024}{9} n_{f}
\end{aligned}
$$




$$
\begin{aligned}
& \left(C_{m^{2}}^{\| \perp}\right)_{2}=0 \\
& \left(C_{m^{2}}^{\| \perp}\right)_{3}=3840+768 \pi^{2}-\frac{128}{3} \pi^{4}-\frac{512}{3} n_{f}, \\
& \left(C_{m^{4}, \mathrm{di}}^{\perp \perp}\right)_{2}=-\frac{28}{3} \text {, } \\
& \left(C_{m^{4}, \mathrm{di}}^{\perp \perp}\right)_{3}=\frac{3650}{27}-\frac{176}{9} \pi^{2}-\frac{16}{135} \pi^{4}-\frac{4384}{9} \zeta_{3}+n_{f}\left(-\frac{412}{9}+\frac{128}{3} \zeta_{3}\right), \\
& \left(C_{m^{4}, \mathrm{di}}^{\| \perp}\right)_{2}=\frac{20}{3}, \\
& \left(C_{m^{4}, \mathrm{di}}^{\| \perp}\right)_{3}=-\frac{11566}{27}+\frac{800}{9} \pi^{2}-\frac{16}{135} \pi^{4}-\frac{6880}{9} \zeta_{3}+n_{f}\left(-\frac{68}{3}+\frac{128}{3} \zeta_{3}\right), \\
& \left(C_{m^{4}, \text { nd }}^{\perp \perp}\right)_{2}=0 \text {, } \\
& \left(C_{m^{4}, \text { nd }}^{\perp \perp}\right)_{3}=\frac{32}{9} \text {, } \\
& \left(C_{m^{4}, \text { nd }}^{\| \perp}\right)_{2}=0 \\
& \left(C_{m^{4}, \mathrm{nd}}^{\| \perp}\right)_{3}=-\frac{544}{9} \text {, } \\
& \left(C_{2}^{\perp \perp}\right)_{1}=-\frac{8}{9}, \\
& \left(C_{2}^{\perp \perp}\right)_{2}=-\frac{175}{27}-\frac{52}{9} \pi^{2}-2 n_{f}, \\
& \left(C_{2}^{\perp \perp}\right)_{3}=-\frac{36190}{81}+\frac{56222}{243} \pi^{2}-\frac{346}{15} \pi^{4}+\frac{8996}{27} \zeta_{3}-\frac{848}{3} \pi^{2} \zeta_{3}+1400 \zeta_{5} \\
& +n_{f}\left(-\frac{7654}{243}+\frac{8}{5} \pi^{4}-\frac{928}{9} \zeta_{3}\right)+n_{f}^{2}\left(-\frac{1198}{729}+\frac{32}{9} \zeta_{3}\right), \\
& \left(C_{2}^{\| \perp}\right)_{1}=\frac{4}{9} \\
& \left(C_{2}^{\| \perp}\right)_{2}=\frac{725}{27}-\frac{28}{3} \pi^{2}-\frac{62}{27} n_{f}, \\
& \left(C_{2}^{\| \perp}\right)_{3}=\frac{176317}{81}-\frac{235214}{243} \pi^{2}-\frac{3254}{81} \pi^{4}+4620 \zeta_{3}+\frac{1904}{3} \pi^{2} \zeta_{3}-3320 \zeta_{5} \\
& +n_{f}\left(-\frac{54658}{243}+24 \pi^{2}+\frac{8}{5} \pi^{4}-\frac{2176}{9} \zeta_{3}\right)+n_{f}^{2}\left(\frac{458}{729}+\frac{32}{9} \zeta_{3}\right), \\
& \left(C_{1}^{\perp \perp}\right)_{1}=\frac{27}{2}, \\
& \left(C_{1}^{\perp \perp}\right)_{2}=\frac{5737}{12}+54 \pi^{2}-144 \zeta_{3}-\frac{160}{9} n_{f}, \\
& \left(C_{1}^{\perp \perp}\right)_{3}=\frac{755000}{27}+\frac{4295}{2} \pi^{2}+\frac{3436}{15} \pi^{4}-16176 \zeta_{3}-1176 \pi^{2} \zeta_{3}+1800 \zeta_{5} \\
& +n_{f}\left(-\frac{321653}{108}-\frac{866}{9} \pi^{2}-\frac{848}{45} \pi^{4}+\frac{20446}{27} \zeta_{3}-16 \pi^{2} \zeta_{3}+600 \zeta_{5}\right) \\
& +n_{f}^{2}\left(\frac{5542}{81}-\frac{16}{3} \zeta_{3}\right)
\end{aligned}
$$




$$
\begin{aligned}
\left(C_{1}^{\| \perp}\right)_{1}= & \frac{21}{2}, \\
\left(C_{1}^{\| \perp}\right)_{2}= & \frac{1321}{4}+61 \pi^{2}-144 \zeta_{3}-\frac{41}{3} n_{f}, \\
\left(C_{1}^{\| \perp}\right)_{3}= & \frac{69761}{3}+\frac{9125}{3} \pi^{2}+\frac{1242}{5} \pi^{4}-18168 \zeta_{3}-1968 \pi^{2} \zeta_{3}+6120 \zeta_{5} \\
& +n_{f}\left(-\frac{820793}{324}-\frac{1240}{9} \pi^{2}-\frac{928}{45} \pi^{4}+\frac{21082}{27} \zeta_{3}-16 \pi^{2} \zeta_{3}+600 \zeta_{5}\right) \\
& +\frac{15025}{243} n_{f}^{2} .
\end{aligned}
$$

Numerically, for $n_{f}=3$, we obtain

$$
\begin{aligned}
& \left(C_{m^{2}}^{\perp \perp}\right)_{n_{f}=3}=8 a^{2}\left[1+a\left(13.656+10.000 L_{z}\right)\right], \\
& \left(C_{m^{2}}^{\| \perp}\right)_{n_{f}=3}=105.496 a^{3}, \\
& \left(C_{m^{4}, \mathrm{di}}^{\perp \perp}\right)_{n_{f}=3}=-\frac{7}{12} a^{2}\left[1-3.429 L_{z}+3.429 L_{z}^{2}\right. \\
& \left.+a\left(17.099-41.523 L_{z}+13.000 L_{z}^{2}+19.429 L_{z}^{3}\right)\right], \\
& \left(C_{m^{4}, \mathrm{di}}^{\| \perp}\right)_{n_{f}=3}=\frac{5}{12} a^{2}\left[1-4.800 L_{z}^{2}+a\left(-14.837+89.515 L_{z}-62.600 L_{z}^{2}-41.600 L_{z}^{3}\right)\right], \\
& \left(C_{m^{4}, \mathrm{nd}}^{\perp \perp}\right)_{n_{f}=3}=a^{3}\left(0.056-2.000 L_{z}\right) \text {, } \\
& \left(C_{m^{4}, \text { nd }}^{\| \perp}\right)_{n_{f}=3}=a^{3}\left(-0.944-2.000 L_{z}\right) \text {, } \\
& \left(C_{2}^{\perp \perp}\right)_{n_{f}=3}=-\frac{2}{9} a\left[1-3.000 L_{z}+a\left(19.549-26.875 L_{z}-4.500 L_{z}^{2}\right)\right. \\
& \left.+a^{2}\left(132.651-104.864 L_{z}-155.226 L_{z}^{2}-13.500 L_{z}^{3}\right)\right], \\
& \left(C_{2}^{\| \perp}\right)_{n_{f}=3}=\frac{1}{9} a\left[1+6.000 L_{z}+a\left(-40.586+70.250 L_{z}+27.000 L_{z}^{2}\right)\right. \\
& \left.+a^{2}\left(-277.555+95.216 L_{z}+622.451 L_{z}^{2}+121.500 L_{z}^{3}\right)\right], \\
& \left(C_{1}^{\perp \perp}\right)_{n_{f}=3}=1+a\left(3.375-3.000 L_{z}\right)+a^{2}\left(49.038+6.617 L_{z}-2.250 L_{z}^{2}\right) \\
& +a^{3}\left(425.570+350.427 L_{z}+5.519 L_{z}^{2}-4.500 L_{z}^{3}\right), \\
& \left(C_{1}^{\| \perp}\right)_{n_{f}=3}=1+2.625 a+a^{2}\left(44.887+18.617 L_{z}\right) \\
& +a^{3}\left(399.885+494.172 L_{z}+83.776 L_{z}^{2}\right) .
\end{aligned}
$$




\section{$5 \quad$ RG improvements of $\Pi_{\perp \perp}^{0}$ and $\Pi_{\| \perp}^{0}$}

In general, a multiplicatively renormalizable structure function $\Pi$ depends on both a renormalization prescription, or scheme, and a renormalization scale $\mu$. It is convenient to deal with the scheme and scale invariant version of $\Pi$, which we denote as $\hat{\Pi}$. Given the RG equation for $\Pi$,

$$
\mu^{2} \frac{\mathrm{d}}{\mathrm{d} \mu^{2}} \Pi(a, \mu) \equiv\left(\mu^{2} \frac{\partial}{\partial \mu^{2}}+\beta(a) a \frac{\partial}{\partial a}\right) \Pi(a, \mu)=\gamma(a) \Pi(a, \mu),
$$

a formal solution for $\hat{\Pi}$ reads

$$
\hat{\Pi}(a)=\frac{\Pi(a, \mu)}{f(a)}, \quad f(a)=\exp \int^{a} \frac{\mathrm{d} x}{x} \frac{\gamma(x)}{\beta(x)} .
$$

Through the order of interest here, we have

$$
\begin{aligned}
f(a)=(a)^{\bar{\gamma}_{1}}\{1 & +\left(\bar{\gamma}_{2}-\bar{\beta}_{2} \bar{\gamma}_{1}\right) a \\
& \left.+\frac{1}{2}\left[\left(\bar{\gamma}_{2}-\bar{\beta}_{2} \bar{\gamma}_{1}\right)^{2}+\bar{\gamma}_{3}+\bar{\beta}_{2}^{2} \bar{\gamma}_{1}-\bar{\beta}_{2} \bar{\gamma}_{2}-\bar{\beta}_{3} \bar{\gamma}_{1}\right] a^{2}+\mathcal{O}\left(a^{3}\right)\right\},
\end{aligned}
$$

where $\bar{\gamma}_{i}=\gamma_{i} / \beta_{1}, \bar{\beta}_{i}=\beta_{i} / \beta_{1}(i=1,2,3)$, and the coefficients $\beta_{i}$ of the beta function are defined as

$$
\beta(a)=\mu^{2} \frac{\mathrm{d}}{\mathrm{d} \mu^{2}} \ln a=\sum_{n=1}^{\infty} \beta_{n} a^{n},
$$

with $\beta_{1}=-11+\frac{2}{3} n_{f}$, etc.

Our results for the scheme invariant functions $\hat{\Pi}_{\perp \perp}^{0}$ and $\hat{\Pi}_{\| \perp}^{0}$ in position space are given by

$$
\begin{aligned}
& \hat{\Pi}_{\perp \perp}^{0}\left(z>0, v^{2}=-1\right)=\frac{128}{z^{4}} a^{1+6 / \beta_{1}}\left[1+\sum_{n=1}^{2}\left(\hat{F}_{\perp \perp}\right)_{n} a^{n}\right], \\
& \hat{\Pi}_{\| \perp}^{0}\left(z>0, v^{2}=-1\right)=-\frac{128 a}{z^{4}}\left[1+\sum_{n=1}^{2}\left(\hat{F}_{\| \perp}\right)_{n} a^{n}\right] .
\end{aligned}
$$

In phenomenological applications, one usually has $n_{f}=0,2,3$. The CFs in eq. (5.5) then take the values

$$
\begin{aligned}
\left(\hat{F}_{\perp \perp}\right)_{1}^{n_{f}=0}= & \frac{9151}{363}+\frac{56}{11} \pi^{2}+10 L_{z}, \\
\left(\hat{F}_{\perp \perp}\right)_{2}^{n_{f}=0}= & \frac{265666457}{263538}+\frac{808070}{3993} \pi^{2}+\frac{798}{121} \pi^{4}-\frac{10206}{11} \zeta_{3} \\
& +L_{z}\left(\frac{326492}{363}+\frac{1792}{11} \pi^{2}\right)+160 L_{z}^{2}, \\
\left(\hat{F}_{\perp \perp}\right)_{1}^{n_{f}=2}= & \frac{190177}{7569}+\frac{152}{29} \pi^{2}+\frac{22}{3} L_{z},
\end{aligned}
$$




$$
\begin{aligned}
& \left(\hat{F}_{\perp \perp}\right)_{2}^{n_{f}=2}=\frac{102303720467}{114579522}+\frac{45401582}{219501} \pi^{2}+\frac{6042}{841} \pi^{4}-\frac{71998}{87} \zeta_{3} \\
& +L_{z}\left(\frac{16534820}{22707}+\frac{12160}{87} \pi^{2}\right)+\frac{880}{9} L_{z}^{2}, \\
& \left(\hat{F}_{\perp \perp}\right)_{1}^{n_{f}=3}=\frac{677}{27}+\frac{16}{3} \pi^{2}+6 L_{z}, \\
& \left(\hat{F}_{\perp \perp}\right)_{2}^{n_{f}=3}=\frac{1216447}{1458}+\frac{16982}{81} \pi^{2}+\frac{68}{9} \pi^{4}-\frac{2330}{3} \zeta_{3} \\
& +L_{z}\left(\frac{5800}{9}+128 \pi^{2}\right)+72 L_{z}^{2}, \\
& \left(\hat{F}_{\| \perp}\right)_{1}^{n_{f}=0}=\frac{28}{3}+\frac{56}{11} \pi^{2}+22 L_{z}, \\
& \left(\hat{F}_{\| \perp}\right)_{2}^{n_{f}=0}=\frac{9011}{18}+\frac{23299}{363} \pi^{2}+\frac{798}{121} \pi^{4}-1026 \zeta_{3} \\
& +L_{z}\left(\frac{1844}{3}+224 \pi^{2}\right)+484 L_{z}^{2} \text {, } \\
& \left(\hat{F}_{\| \perp}\right)_{1}^{n_{f}=2}=\frac{88}{9}+\frac{152}{29} \pi^{2}+\frac{58}{3} L_{z}, \\
& \left(\hat{F}_{\| \perp}\right)_{2}^{n_{f}=2}=\frac{74591}{162}+\frac{567709}{7569} \pi^{2}+\frac{6042}{841} \pi^{4}-\frac{2798}{3} \zeta_{3} \\
& +L_{z}\left(\frac{14348}{27}+\frac{608}{3} \pi^{2}\right)+\frac{3364}{9} L_{z}^{2}, \\
& \left(\hat{F}_{\| \perp}\right)_{1}^{n_{f}=3}=10+\frac{16}{3} \pi^{2}+18 L_{z}, \\
& \left(\hat{F}_{\| \perp}\right)_{2}^{n_{f}=3}=\frac{2627}{6}+\frac{2185}{27} \pi^{2}+\frac{68}{9} \pi^{4}-886 \zeta_{3} \\
& +L_{z}\left(488+192 \pi^{2}\right)+324 L_{z}^{2} \text {. }
\end{aligned}
$$

Numerically, the same results read

$$
\begin{aligned}
& \left(\hat{F}_{\perp \perp}\right)_{n_{f}=0}=1+a\left(18.864+2.500 L_{z}\right)+a^{2}\left(158.283+156.705 L_{z}+10.000 L_{z}^{2}\right) \\
& \left(\hat{F}_{\perp \perp}\right)_{n_{f}=2}=1+a\left(19.214+1.833 L_{z}\right)+a^{2}\left(164.958+131.729 L_{z}+6.111 L_{z}^{2}\right) \\
& \left(\hat{F}_{\perp \perp}\right)_{n_{f}=3}=1+a\left(19.428+1.500 L_{z}\right)+a^{2}\left(169.120+119.235 L_{z}+4.500 L_{z}^{2}\right) \\
& \left(\hat{F}_{\| \perp}\right)_{n_{f}=0}=1+a\left(14.895+5.500 L_{z}\right)+a^{2}\left(33.950+176.591 L_{z}+30.250 L_{z}^{2}\right) \\
& \left(\hat{F}_{\| \perp}\right)_{n_{f}=2}=1+a\left(15.377+4.833 L_{z}\right)+a^{2}\left(48.713+158.228 L_{z}+23.361 L_{z}^{2}\right) \\
& \left(\hat{F}_{\| \perp}\right)_{n_{f}=3}=1+a\left(15.659+4.500 L_{z}\right)+a^{2}\left(56.719+148.935 L_{z}+20.250 L_{z}^{2}\right) .
\end{aligned}
$$




\section{RG improvements of the CFs}

The $\mathcal{O}\left(1 / z^{4}\right)$ and $\mathcal{O}\left(1 / z^{2}\right)$ terms in eq. (2.6),

$$
\Pi_{\perp \perp}^{m^{2}}(z)=\frac{C_{0}^{\perp \perp}(z)}{z^{4}}+\frac{C_{m^{2}}^{\perp \perp}(z)}{z^{2}} \sum_{i} m_{i}^{2}
$$

satisfy a simple evolution equation of the form ${ }^{2}$

$$
\mu^{2} \frac{d}{d \mu^{2}} \Pi_{\perp \perp}^{m^{2}}(z)=\gamma_{\perp \perp}(a) \Pi_{\perp \perp}^{m^{2}}(z)
$$

where

$$
\mu^{2} \frac{d}{d \mu^{2}} \equiv \mu^{2} \frac{\partial}{\partial \mu^{2}}+\beta(a) a \frac{\partial}{\partial a}+\gamma_{m}(a) m_{i} \frac{\partial}{\partial m_{i}},
$$

with $\gamma_{m}(a)=-4 a+\left(-\frac{202}{3}+\frac{20}{9} n_{f}\right) a^{2}+\cdots$ being the quark mass anomalous dimension.

The evolution equations for the remaining CFs are more complicated due to mixing between the operators $O_{1}, O_{2}$ and combinations quartic in quark masses [33, 36, 37]. The mixing is described by

$$
\begin{aligned}
& \mu^{2} \frac{d}{d \mu^{2}} O_{1}=\gamma_{11} O_{1}+\gamma_{12} O_{2}+4 a \frac{\partial}{\partial a} \gamma_{0}, \\
& \mu^{2} \frac{d}{d \mu^{2}} O_{2}=-4 \gamma_{0}
\end{aligned}
$$

where

$$
\gamma_{11}=-a \frac{\partial}{\partial a} \beta, \quad \gamma_{12}=4 a \frac{\partial}{\partial a} \gamma_{m},
$$

and $\gamma_{0}$ is the anomalous dimension of the vacuum energy [37-39],

$$
\gamma_{0}(a, m)=\gamma_{0}^{\mathrm{di}}(a) \sum_{i} m_{i}^{4}+\gamma_{0}^{\mathrm{nd}}(a) \sum_{i \neq j} m_{i}^{2} m_{j}^{2},
$$

with

$$
\begin{aligned}
\gamma_{0}^{\mathrm{di}}(a) & =-\frac{3}{16 \pi^{2}}\left[1+\frac{16}{3} a+\left(\frac{626}{9}-\frac{32}{3} \zeta(3)-\frac{20}{3} n_{f}\right) a^{2}\right], \\
\gamma_{0}^{\mathrm{nd}}(a) & =\frac{6}{\pi^{2}} a^{2} .
\end{aligned}
$$

The resulting evolution equations for the $\mathrm{CFs} \mathbb{C}_{\mathbb{1}} \equiv \frac{g^{2}}{12} C_{1}, C_{2}, C_{m^{4}, \mathrm{di}}$, and $C_{m^{4}, \text { nd }}$ read:

$$
\begin{aligned}
\mu^{2} \frac{d}{d \mu^{2}} \mathbb{C}_{1}^{\perp \perp} & =\left(\gamma_{\perp \perp}-\gamma_{11}\right) \mathbb{C}_{1}^{\perp \perp}, \\
\mu^{2} \frac{d}{d \mu^{2}} C_{2}^{\perp \perp} & =\gamma_{\perp \perp} C_{2}^{\perp \perp}-\gamma_{12} \mathbb{C}_{1}^{\perp \perp}, \\
\mu^{2} \frac{d}{d \mu^{2}} C_{m^{4}, \mathrm{di}}^{\perp \perp} & =\left(\gamma_{\perp \perp}-4 \gamma_{m}\right) C_{m^{4}, \mathrm{di}}^{\perp \perp}-\left(4 a \frac{\partial}{\partial a} \gamma_{0}^{\mathrm{di}}\right) \mathbb{C}_{1}^{\perp \perp}+4 C_{2}^{\perp \perp} \gamma_{0}^{\mathrm{di}}, \\
\mu^{2} \frac{d}{d \mu^{2}} C_{m^{4}, \text { nd }}^{\perp \perp} & =\left(\gamma_{\perp \perp}-4 \gamma_{m}\right) C_{m^{4}, \text { nd }}^{\perp \perp}-\left(4 a \frac{\partial}{\partial a} \gamma_{0}^{\text {nd }}\right) \mathbb{C}_{1}^{\perp \perp}+4 C_{2}^{\perp \perp} \gamma_{0}^{\text {nd }} .
\end{aligned}
$$

We checked ${ }^{3}$ that our results do satisfy eqs. (6.2) and (6.8).

\footnotetext{
${ }^{2}$ In this section, we only consider CFs corresponding to the invariant function $\Pi_{\perp \perp}$. The corresponding relations for $\Pi_{\| \perp}$ emerge by replacing $\perp \perp$ with $\| \perp$.

${ }^{3}$ In addition to the ADs $\gamma_{\perp \perp}, \gamma_{\| \perp}$, and $\gamma_{0}$, we have used the three-loop $\beta$ function [40, 41] and the two-loop quark mass anomalous dimension $\gamma_{m}[42,43]$.
} 


\section{Conclusions}

We considered the non-local operator consisting of two gluon field strength tensors connected by a straight Wilson line and studied its VEV, which is determined by two nonperturbative Lorentz scalar functions. For each of the latter, we performed an OPE through mass dimension four, which, besides a purely perturbative structure function, involves the VEVs of three local operators, and calculated the $\mathrm{AD}$ of the structure function and the CFs of the three local operators through three loops, order $a^{3}$, in the modified minimalsubtraction $(\overline{\mathrm{MS}})$ scheme. In our recent work [1], the structure functions were derived through three loops and the ADs through two loops. Using the ADs, we also performed a RG improvement of the structure functions and presented their renormalization scheme invariant counterparts thus resulting, again through three loops. These results should be of interest to the lattice QCD community in studies of the QCD vacuum structure and serve as normalization factors in calculations of gluon PDFs within the qPDF approach, eliminating the need for nonperturbative subtractions of linear divergences.

\section{Acknowledgments}

The work of V.M.B. and B.A.K. was supported in part by DFG Research Unit FOR 2926 under Grant No. 409651613. The work of K.G.C. was supported by DFG Grant No. CH 1479/2-1.

Open Access. This article is distributed under the terms of the Creative Commons Attribution License (CC-BY 4.0), which permits any use, distribution and reproduction in any medium, provided the original author(s) and source are credited.

\section{References}

[1] V.M. Braun, K.G. Chetyrkin and B.A. Kniehl, Renormalization of parton quasi-distributions beyond the leading order: spacelike vs. timelike, JHEP 07 (2020) 161 [arXiv:2004.01043] [INSPIRE].

[2] H.G. Dosch, Nonperturbative methods in quantum chromodynamics, Prog. Part. Nucl. Phys. 33 (1994) 121 [INSPIRE].

[3] A. Di Giacomo, H.G. Dosch, V.I. Shevchenko and Y.A. Simonov, Field correlators in QCD: Theory and applications, Phys. Rept. 372 (2002) 319 [hep-ph/0007223] [INSPIRE].

[4] D. Gromes, Space-time Dependence of the Gluon Condensate Correlation Function and Quarkonium Spectra, Phys. Lett. B 115 (1982) 482 [InSPIRE].

[5] I.I. Balitsky, Wilson loop for the stretched contours in vacuum fields and the small-distance behaviour of the interquark potential, Nucl. Phys. B 254 (1985) 166 [INSPIRE].

[6] M. Campostrini, A. Di Giacomo and Š. Olejník, On the possibility of detecting gluon condensation from the spectra of heavy quarkonia, Z. Phys. C 31 (1986) 577 [inSPIRE].

[7] Y.A. Simonov, S. Titard and F.J. Yndurain, Heavy quarkonium systems and nonperturbative field correlators, Phys. Lett. B 354 (1995) 435 [hep-ph/9504273] [InSPIRE]. 
[8] N. Brambilla, A. Pineda, J. Soto and A. Vairo, Potential NRQCD: an effective theory for heavy quarkonium, Nucl. Phys. B 566 (2000) 275 [hep-ph/9907240] [INSPIRE].

[9] N. Brambilla, X. Garcia i Tormo, J. Soto and A. Vairo, The logarithmic contribution to the QCD static energy at $N^{4} L O$, Phys. Lett. B 647 (2007) 185 [hep-ph/0610143] [INSPIRE].

[10] N. Brambilla, H.S. Chung and A. Vairo, Inclusive Hadroproduction of P-Wave Heavy Quarkonia in Potential Nonrelativistic QCD, Phys. Rev. Lett. 126 (2021) 082003 [arXiv: 2007.07613] [INSPIRE].

[11] N. Brambilla, H.S. Chung, D. Müller and A. Vairo, Decay and electromagnetic production of strongly coupled quarkonia in pNRQCD, JHEP 04 (2020) 095 [arXiv: 2002. 07462] [INSPIRE].

[12] A. Di Giacomo and H. Panagopoulos, Field strength correlations in the QCD vacuum, Phys. Lett. B 285 (1992) 133 [INSPIRE].

[13] M. D'Elia, A. Di Giacomo and E. Meggiolaro, Field strength correlators in full QCD, Phys. Lett. B 408 (1997) 315 [hep-lat/9705032] [INSPIRE].

[14] G.S. Bali, N. Brambilla and A. Vairo, A Lattice determination of QCD field strength correlators, Phys. Lett. B 421 (1998) 265 [hep-lat/9709079] [INSPIRE].

[15] TUMQCD collaboration, Determination of the QCD coupling from the static energy and the free energy, Phys. Rev. D 100 (2019) 114511 [arXiv:1907.11747] [INSPIRE].

[16] M. D'Elia, A. Di Giacomo and E. Meggiolaro, Gauge invariant field strength correlators in pure Yang-Mills and full QCD at finite temperature, Phys. Rev. D 67 (2003) 114504 [hep-lat/0205018] [INSPIRE].

[17] A.A. Vladimirov, Self-Contained Definition of the Collins-Soper kernel, Phys. Rev. Lett. 125 (2020) 192002 [arXiv: 2003. 02288] [INSPIRE].

[18] X. Ji, Y. Liu, Y.-S. Liu, J.-H. Zhang and Y. Zhao, Large-Momentum Effective Theory, arXiv:2004.03543 [INSPIRE].

[19] Z.-Y. Fan, Y.-B. Yang, A. Anthony, H.-W. Lin and K.-F. Liu, Gluon Quasi-Parton-Distribution Functions from Lattice QCD, Phys. Rev. Lett. 121 (2018) 242001 [arXiv: 1808.02077] [INSPIRE].

[20] W. Wang, S. Zhao and R. Zhu, Gluon quasidistribution function at one loop, Eur. Phys. J. C 78 (2018) 147 [arXiv: 1708.02458] [INSPIRE].

[21] W. Wang, J.-H. Zhang, S. Zhao and R. Zhu, Complete matching for quasidistribution functions in large momentum effective theory, Phys. Rev. D 100 (2019) 074509 [arXiv: 1904.00978] [INSPIRE].

[22] I. Balitsky, W. Morris and A. Radyushkin, Gluon pseudo-distributions at short distances: Forward case, Phys. Lett. B 808 (2020) 135621 [arXiv:1910.13963] [INSPIRE].

[23] H. Dorn and E. Wieczorek, Renormalization and short distance properties of string type equations in QCD, Z. Phys. C 9 (1981) 49 [Erratum ibid. 9 (1981) 274] [INSPIRE].

[24] H. Dorn, D. Robaschik and E. Wieczorek, Renormalization and Short Distance Properties of Gauge Invariant Gluoinum and Hadron Operators, Annalen Phys. 40 (1983) 166.

[25] V.M. Braun, A. Vladimirov and J.-H. Zhang, Power corrections and renormalons in parton quasidistributions, Phys. Rev. D 99 (2019) 014013 [arXiv: 1810.00048] [InSPIRE].

[26] V.S. Dotsenko and S.N. Vergeles, Renormalizability of phase factors in non-abelian gauge theory, Nucl. Phys. B 169 (1980) 527 [INSPIRE]. 
[27] A.G. Grozin, Methods of Calculation of Higher Power Corrections in QCD, Int. J. Mod. Phys. A 10 (1995) 3497 [hep-ph/9412238] [INSPIRE].

[28] M. Eidemuller and M. Jamin, QCD field strength correlator at the next-to-leading order, Phys. Lett. B 416 (1998) 415 [hep-ph/9709419] [INSPIRE].

[29] S.G. Gorishnii, S.A. Larin and F.V. Tkachov, The algorithm for OPE coefficient functions in the MS scheme, Phys. Lett. B 124 (1983) 217 [InSPIRE].

[30] S.G. Gorishnii and S.A. Larin, Coefficient functions of asymptotic operator expansions in minimal subtraction scheme, Nucl. Phys. B 283 (1987) 452 [INSPIRE].

[31] J.A.M. Vermaseren, New features of FORM, math-ph/0010025 [INSPIRE].

[32] T. van Ritbergen, A.N. Schellekens and J.A.M. Vermaseren, Group theory factors for Feynman diagrams, Int. J. Mod. Phys. A 14 (1999) 41 [hep-ph/9802376] [InSPIRE].

[33] H. Kluberg-Stern and J.B. Zuber, Ward identities and some clues to the renormalization of gauge-invariant operators, Phys. Rev. D 12 (1975) 467 [INSPIRE].

[34] J.C. Collins, A. Duncan and S.D. Joglekar, Trace and dilatation anomalies in gauge theories, Phys. Rev. D 16 (1977) 438 [INSPIRE].

[35] N.K. Nielsen, The energy-momentum tensor in a non-Abelian quark gluon theory, Nucl. Phys. B 120 (1977) 212 [INSPIRE].

[36] V. Spiridonov, Anomalous Dimension of $G_{\mu \nu}^{2}$ and $\beta$ Function, (1984) IYaI-P-0378.

[37] V.P. Spiridonov and K.G. Chetyrkin, Nonleading mass corrections and renormalization of the operators $m \bar{\psi} \psi$ and $G_{\mu \nu}^{2}$, Yad. Fiz. 47 (1988) 81 [Sov. J. Nucl. Phys. 47 (1988) 522] [INSPIRE].

[38] K.G. Chetyrkin and J.H. Kühn, Quartic mass corrections to $R_{\text {had }}$, Nucl. Phys. B 432 (1994) 337 [hep-ph/9406299] [inSPIRE].

[39] P.A. Baikov and K.G. Chetyrkin, QCD vacuum energy in 5 loops, PoS RADCOR2017 (2018) 025 [INSPIRE].

[40] O.V. Tarasov, A.A. Vladimirov and A.Y. Zharkov, The Gell-Mann-Low Function of QCD in the Three Loop Approximation, Phys. Lett. B 93 (1980) 429 [INSPIRE].

[41] S.A. Larin and J.A.M. Vermaseren, The three-loop QCD $\beta$-function and anomalous dimensions, Phys. Lett. B 303 (1993) 334 [hep-ph/9302208] [INSPIRE].

[42] R. Tarrach, The Pole Mass in Perturbative QCD, Nucl. Phys. B 183 (1981) 384 [InSPIRE].

[43] O. Nachtmann and W. Wetzel, The $\beta$-function for Effective Quark Masses to Two Loops in QCD, Nucl. Phys. B 187 (1981) 333 [InSPIRE]. 